\title{
METHODOLOGIES FOR MANAGING COMPLEX SYSTEMS UNDER UNCERTAINTY
}

\author{
LAZAR D. GITELMAN, TATYANA B. GAVRILOVA \& MIKHAIL V. KOZHEVNIKOV \\ Ural Federal University, Russia
}

\begin{abstract}
The problems of managing socio-technical systems, the complexity of which is constantly increasing, in a time when the external environment becomes more and more volatile, and large-scale changes develop unpredictable effects, requires managers to have an adequate response and act pre-emptively in unexpected situations. In this regard, it appears practical to apply methods of systems engineering that was created for designing complex systems and keeping them functional throughout their lifecycle. An analysis of various theoretical concepts and practical approaches employed in systems engineering showed their potential for being adapted to the purposes of managing complex systems under uncertainty. For example, the method of engineering resilience could be used for training interdisciplinary teams that need to be able to handle complex problems with speed, coordination, initiative and mutual support. It also appears usable for setting up coordination centers that produce strategic assessments of implications, set priorities, distribute resources, motivate teams and provide training, thus building preparedness to address unforeseen problems. The method of Agile systems engineering is effective for pre-empting problems that occur as a result of intensive technological development and are characterized by capriciousness, uncertainty, risk, variability, and evolution. The above methods were applied for training breakthrough teams - an original project of the authors that has proved highly efficient for solving tasks of high complexity and uncertainty in energy companies and at Ural Federal University.
\end{abstract}

Keywords: complex systems, systems engineering, uncertainty, resilience, Agile systems, interdisciplinarity, breakthrough team.

\section{INTRODUCTION}

The explosive adoption of new technologies, a qualitative increase in the complexity of technical and socio-technical systems that are being built on the basis of these technologies, the penetration of artificial intelligence into technological chains, the development of computer integrated manufacturing and digitalization, and an array of other innovations determine a snowballing growth in new problems that are a lot more complex and require new solution tools both in engineering and management that utilize new knowledge and a large amount of information.

A problem that is becoming particularly urgent has to do with the fact that complex systems have not taken a finished shape (do not have an ultimate set of solutions) and must be regularly upgraded to change in line with new requirements. Generally speaking, this problem has existed before in systems engineering, but today's uncertainty and the dynamics of change, even chaotic to a certain degree, makes this problem particularly relevant and significant. In such conditions, systems must maintain their resilience through the development of agility and adaptability, that is, through the consideration of factors of future uncertainty. That's why, systems are equipped with the capability to change (transform) quickly that is built into them, and capacity for proactive actions by means of:

- building various reserves in case of unforeseen circumstances;

- constant monitoring of weak signals and digital indicators;

- modular construction and infrastructure that provides for urgent replacements (of one module for another). 
All of the above requires additional spending and increases the cost of the system. This, however, is outweighed by resulting benefits: agility, reliability, the option of the quick replacement (reset) of manufacturing processes. These are the parameters that are of paramount importance to government institutions, business, and, of course, universities that are faced with a new imperative - that of anticipatory learning.

Using competences that have been mastered in advance one can identify and define what is going on outside the system, assess the prospects of change and select the necessary tools. There was a practical need for a methodology that would reduce the risks and influence of systemic mistakes and ensure collaboration on the boundaries of disciplines where unforeseen problems arise most often. Systems engineering became the source of such a methodology [1] as it presents a way of tackling the complexity of systems being designed.

Systems engineering is defined as a discipline that provides the groundwork and describes the key principles of managing the creation of systems of any size and designation, develops the methodology and tools for managing the lifecycle of complex systems, creates the principles and instruments for their development. The essence of systems engineering as a research and technical discipline lies in a holistic lifecycle-based approach that implies a creative interwoven combination of technical, managerial and economic achievements and contributions from other fields [2]. Moreover, systems engineering is an interdisciplinary approach governing the total technical and managerial effort required to transform a set of stakeholder needs, expectations, and constraints into a solution, and to support that solution throughout its life [3].

Today, systems engineering is faced with serious challenges that require a response. It has become clear that the spectrum of social needs that must been taken into account when constructing engineering and technical systems plays an increasingly crucial role in their behavior and stability. The systems by themselves are getting integrated into larger sociotechnical and economic systems that require synchronized action of all their versatile components for the sake of common goals.

It is obvious that problems associated with the study of the multifaceted complexity of systems are at the center of today's agenda.

1. The complexity of objects increases faster than the capacity to manage it, which results in higher risks of inadequate specification and incomplete verification of functional capabilities.

2. Systems design is largely determined by emerging capabilities that apply to its separate components rather than by general systems architecture principles. As a result, the system lacks strength, is hard to test, too complex and too expensive to run.

3. Problems on the borderlines between the phases of the project lifecycle (knowledge and investment are lost in transition to the next phase and are not inherited by other projects) lead to higher risks and costs of design and operation due to the delayed detection of design defects.

4. Technical and management activities within one project are poorly connected, preventing effective decision making.

5. The majority of major disasters were caused by mistakes in the understanding and evaluation of risks that emerge as a result of interaction of single units of the system (systemic risks); they cannot be detected through the study of reliability and vulnerability of single components.

The problems listed above generate a cascade of challenges and bring to the forefront two key tasks that are addressed by this study: 
- creation of mechanisms that would ensure the stable development of complex systems;

- development of competencies of managerial workers and formation of cross-disciplinary teams that have a holistic vision of the future and are engaged in large-scale projects of digital transformation (breakthrough) in an evolving environment.

\section{CHARACTERISTICS OF SYSTEMS THAT ARE FIT FOR FUNCTION IN A TURBULENT ENVIRONMENT}

Research and development of systems engineering processes and methods look into the problems of coherence, system processes, systems pathology, human-machine interaction in systems control, modeling of systems behavior and development. One of the top issues for research states that the complexity of objects increases faster than the capacity to control it, which results in higher risks of inadequate specification and incomplete verification of functional capabilities. Numerous new elements of systems are emerging in the count of millions. To represent them in various aspects (engineering and technical, information, organizational, social ones) all kinds of models are required. It is obvious that design, production, operation and optimization procedures grow unprecedentedly complex, too. Thousands of organizations get engaged in large projects! Coordination of their efforts, balancing of their interests and constantly changing requirements, the use of a common language of concepts pose an extreme management challenge. Maintaining a crossdisciplinary integrity is key to the success of the entire project. It's no coincidence that systems engineering as the methodological framework for such projects is part of the agenda of leading global corporations and a core course on the curriculum of major universities. Another issue that is typically highlighted is a weak connection between technical and managerial activities in projects, which is a barrier to the correct assessment of risks and to the development of effective solutions.

Consequently, the action area of systems engineering and the scope of issues it addresses largely overlaps management issues [4].

Systems that are adapted to operating in a highly unstable (well above the average level) environment and performing critical functions disruptions to which are either unacceptable or too costly require a special approach to design and development. Factors of the external environment that make such an approach necessary include: capriciousness, uncertainty, risk, variation, evolution [5]. The key feature of systems designed this way, or Agile systems, is their ability to succeed in an evolving external environment that is described by uncertainty and unpredictability. This ability is ensured through skillful execution of both reactive and proactive actions.

Reactive actions are usually triggered by external events that provoke a response. This could be the utilization of new opportunities or response to a threat. Such actions are supported by correction, the versatility of components, the expansion of the system's boundaries and changes in its configuration.

Proactive changes are usually launched from within and are aimed at the application of new knowledge (or fulfillment of new opportunities for already accumulated knowledge) to create new values. Proactive actions imply the creation of new components and structures, improvements to the functionality of the existing elements, redistribution of functions and an upgrade of components and connections [6], [7].

The ability of a system to adapt to change is measured in four dimensions: speed, economic performance (an acceptable sum total of costs), predictability and scale. These indicators are used together in a reasonable combination [8].

Architectural patterns for such Agile systems (Agile architecture pattern, AAP) include several critical components: (1) a roster of drag-and-drop modules that realize the target 
functionality; (2) a passive infrastructure with a minimum, yet sufficient set of rules and standards that ensure the connectivity of the modules; and (3) an active infrastructure with specific properties that are required for supporting changes in the structure of the system in the course of its creation and operation [9].

Modules have a well-defined interface which conforms to the passive infrastructure. Each one of them is fully equipped to exercise their functionality and is not dependent on the functional methods of other modules. The passive infrastructure provides smooth and quick connectivity between modules and the system and easy disconnection of modules that are no longer needed by the system. Its value is in isolating the modules so that unexpected side effects are ruled out when the structure of the system changes.

\subsection{Principles of Agile-systems design and behavior}

An Agile system is built with a view to enabling continuous change: their structure can change as often as required by circumstances. For a change to take place without disrupting the system operation the following conditions must be met: (1) the roster of available modules must evolve to be always what is needed; (2) the modules that are needed must always be in deployable condition; (3) situational knowledge enables timely adjustment of the configuration; (4) the assembly of new configurations must be accomplished with the kind of almost automatic perfection; and (5) both the passive and active infrastructures must have evolved when new configurations and new resources require new standards and rules. The active infrastructure that is plugged into the system at the design stage and accommodates effective transformations that are determined by situational knowledge.

Empirical evidence and observations of existing Agile systems in operation made it possible to identify the key design principles that ensure reusability, reconfigurability and scalability (RRS) [9], [10]. The principles can be divided into three groups (Table 1).

Table 1: Agile systems design principles.

\begin{tabular}{|l|l|l|}
\hline Reusable principles & Reconfigurable principles & Scalable principles \\
\hline$\bullet$ Encapsulated & $\bullet$ Peer-peer interaction & $\bullet$ Evolving infrastructure \\
modules & $\bullet$ Distributed control and information & $\begin{array}{l}\bullet \text { Redundancy and } \\
\text { diversity }\end{array}$ \\
$\bullet$ Plug compatibility & $\bullet$ Deferred commitment & $\bullet$ Elastic capacity \\
$\bullet$ Facilitated reuse & $\bullet$ Self-organization & \\
\hline
\end{tabular}

\subsection{Agile systems life cycle model}

A peculiarity of Agile systems is a life cycle with asynchronous and parallel processes at all stages [9], [10]. These processes are triggered and adjusted by application of situational knowledge.

The life cycle management pattern for Agile systems contains three "in-built" systems that function simultaneously and interdependently (Fig. 1), establishing system reference boundaries and subsystem reference boundaries [11].

System 1 (Target System): the subject of design and support the life cycle of which needs to be managed. It is a logical system which results from or is subject to innovation. It is potentially Agile and can easily be replaced with another system (module) with the necessary properties.

System 2 (Target System (and components) Life Cycle Domain System) is a system within which the Target System (and its components) will exist during its life cycle. It is the provider 
of maintenance and life support to the Target System. System 2 includes the external environment of the Target system and all actors the latter interacts with, e.g. its operational domain and all life cycle management subsystems - development, production, integration, maintenance, operations.

System 3 (System of Innovation, SOI) - a logical system that includes System 1 and System 2 , and that is responsible for managing the life cycle of System 2. System 3 also includes management and learning subsystems for System 2, thus supporting its life cycle and quick reaction to change.

The arrows in Fig. 1 indicate information exchange between systems that reduces internal uncertainty and provides regulatory support.

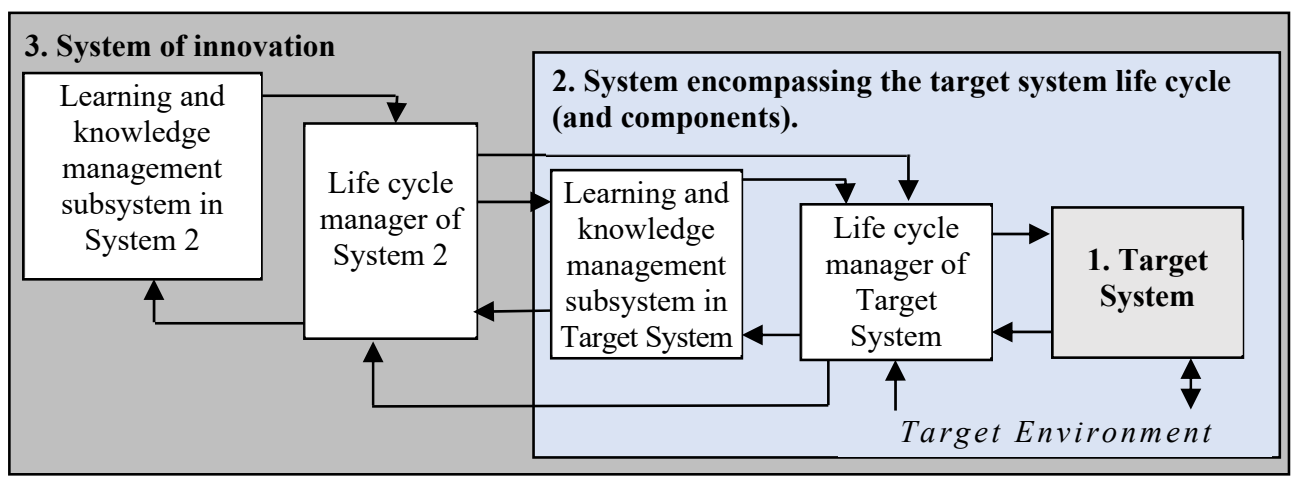

Figure 1: Iconic view of the ASELCM pattern [11].

Differentiating learning subsystems within System 2 and System 3 from other subsystems is necessary for understanding the following aspects of agility:

- management in such systems can be executed only on the basis of knowledge accumulation in all ISO 15288 process areas [12];

- to extract and retain such knowledge an effective learning system is needed.

Learning includes observation, experimental discovery, questioning, noticing differences between what is observed and what is believed, and extracting patterns from instances.

The adaptive capacity and the capacity for development amid uncertainty and unpredictable changes calls for continuous learning, retention and accumulation of learned knowledge and its application in management and further learning.

\section{INCREASING THE RESILIENCE OF SYSTEMS WITH A LONG LIFE-CYCLE}

The branch of systems engineering that designs systems that must continue to have the required functionality for a long time applies tools that make it possible to avoid disasters through preventive action and by increasing the resilience of a system (its recovery after a failure) and its adaptive capacity. Fig. 2 depicts an approach to design of resilient systems that is adopted by systems engineering.

Investigations of the practice of creation and application of resilient systems enabled one to identify their typical characteristics [13] and formulate design principles that need to be adhered to for creating resilient systems [14]. 


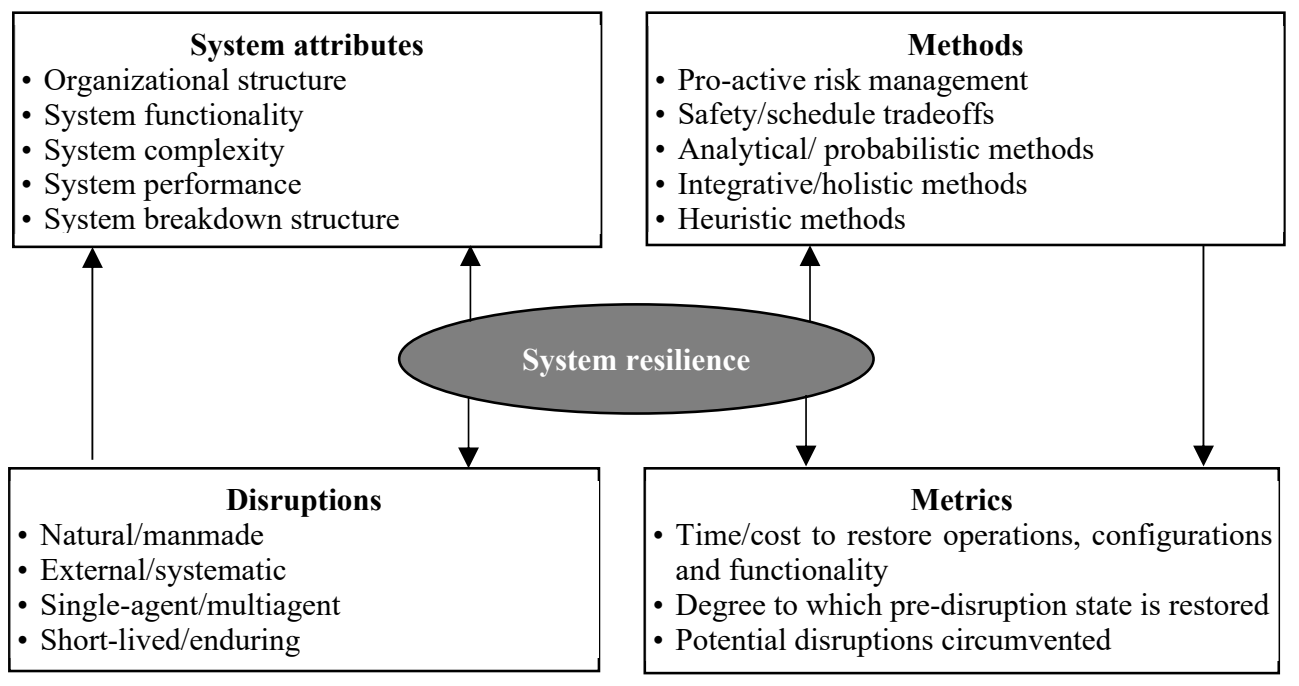

Figure 2: System resilience concept (adapted from [16]).

The indispensable attributes of a resilient system include capacity, flexibility, tolerance, and cohesion [14], [15]. Capacity means the ability of a system to survive in adverse conditions; flexibility is the ability of a system to adapt to a threat; tolerance is the ability of a system to avoid a drastic loss of functionality; cohesion is the ability of a system to act as a unified whole in the face of a threat.

Resilience is not a property (an attribute) of a system, but its behavior (what it can do). Resilience implies that the system can:

- act preemptively, that is, it can anticipate upcoming hazards in order to start adapting beforehand and reduce the risk of decompensation;

- act synchronously, that is, regulate activities at various levels in order to respond timely to fast-changing events and reduce the risk generated by a goal conflict;

- be prepared to act, that is, foster its capability to respond to future "surprises" and reduce the risk of unsustainability;

- learn proactively, that is, learn to understand the signs, causes and sources of unsustainability and improve its capability to counter them (resilient performance) before the occurrence of a major collapse by examining the experience of tracking down and resolving unforeseen situations [15].

The principles of resilient system design are described in great detail in [16]. For each of the key principles, there is an outline of second-tier support principles. Relationships between them and combination options are elaborated on in [17], [18]. The sources also describe examples of the actual application of the principles and their combinations.

\section{NEW CHALLENGES ARE GIVING A NEW SHAPE TO MANAGEMENT}

It is obvious that major changes are about to take place in business management and management standards. The way of thinking that focuses on adaptive and resilient systems forces practitioners to weigh potential threats to system stability and work out countermeasures to prevent long-term losses. At the same time, managers need to be able to 
foresee changes and take advantage of them instead of resisting them and seeking to maintain a state of equilibrium (status quo). This paradigm produces an approach that includes anticipatory learning that incorporates the experience of handling extraordinary situations and efforts aimed at developing the capacity for quick reaction and adaptation.

As the problem is multidisciplinary by nature, finding a solution to it requires that technical and managerial processes are executed as one whole. One also has to take into account the established culture and transform it, if necessary, eradicating negative convictions that undermine the system's resilience. It is necessary to nurture skills such as the ability to realize anomalies, coordinate efforts that involve various functional roles, the ability to stop a potential cascading failure, the ability to decide which infrastructural functions are critical for prevention of destruction and for recovery activities. A manager must grow the capacity of a system to adapt to unforeseen changes, to mitigate uncertainty and improve learning while understanding that future threats will not be a repetition of the past. By practicing response algorithms before a destructive event happens, managers build preparedness to respond to future challenges.

To maintain the critical characteristics of polycentric systems at a level that ensures the integrity of the system that remains resilient and able to change the form of coordination between centers, it is necessary to know how to utilize flexibility reserves. Building, preserving and using such reserves at each level of the system enable its sustainable operation in case of an abruptly occurring hazard.

The key functions of management are, therefore:

- to ensure the normal operation of the polycentric system (autonomy of units, reallocation of resources and powers);

- integration of information and communication domains and adaptive restructuring of the system (network) at each level;

- concurrence (pursuit of a shared goal) and policy of compromise (balancing for adaptation at each level).

Managers need to identify types of complexity in a system and its external environment, pick appropriate approaches and methods, reallocate power and responsibility within the governance system to ensure a timely and adequate reaction to changes coming from the outside, and to train staff members to perform new roles. In collaboration with specialists, they must work on reducing internal complexity to the lowest acceptable level (by changing the structure, processes, governance system), interact with developers and suppliers to control external and internal complexity. For that, the following capabilities, knowledge and competences will be required:

- $\quad$ systems literacy and a general understanding of complex adaptive systems;

- complexity-oriented thinking;

- profound knowledge of technological and organizational peculiarities of the business they work in as well as a good understanding of the capabilities and risks of new technologies;

- the ability to establish effective communication and interaction among professionals in various domains;

- $\quad$ knowledge of Agile-systems and Agile-processes that are used for systems creation and development;

- readiness to learn continuously from crises;

- ability to nurture a culture supporting Agile-systems and resilience in the organization. 
When dealing with complex systems, the key success factor is a strong cross-disciplinary team. Such teams ensure the successful adoption of new technologies in the existing system and the subsequent improvement of processes and creation of additional value for customers.

Large-scale transformations that are constantly generated by the external environment bring about unexpected and unforeseeable effects that one must be prepared to. It is impossible to have an action plan for an unpredictable situation, but it is necessary to have a reaction ready in case of abrupt changes. Teams that are handling the new problem not only need to act fast, but also work in concert, proactively and cooperatively. The coordination center is required to quickly deliver a strategic assessment of consequences, set priorities, allocate resources (reserves), provide motivation (goal balancing) and training for teams to make them prepared for handling unforeseen problems.

Members of such teams not only ensure information exchange and a combination of various points of view on emerging opportunities and threats, but also accumulate and formalize knowledge, work out effective applications and methods of application of the knowledge for addressing routine problems and for battling unforeseen destructive impacts. Such teams are best fitted for proactive learning and sharing lessons learnt from the practice of overcoming systemic stresses (successes and failures).

\section{EXPERIENCE OF TRAINING CROSS-DISCIPLINARY BREAKTHROUGH TEAMS}

A breakthrough team is a cross-disciplinary team of highly qualified specialists (managers, engineers, economists, IT professionals, outside experts) that is capable of powerful contextual perception, can deliver a comprehensive vision of prospects, generate new ideas, and have tools and competences to implement profound transformations within technological modernization, digitalization and development of new business models. Candidates for the team are picked among unique professionals whose personal traits and professional competencies have enabled them handle particularly complex problems with a high degree of novelty and uncertainty: holistic vision of the future, global thinking, entrepreneurial spirit, focus on promising technologies, a partnership mindset.

The key personal traits that are sought after include:

1. a sense of purpose and ambitiousness;

2. constructive result-oriented thinking;

3. ability to bear responsibility for unconventional solutions;

4. preparedness to cooperation, ability to quickly expand one's knowledge and experience.

A breakthrough team that has been trained to handle unique projects possesses competences that are shown in Fig. 3.

Team work aiming for a breakthrough prioritizes the creation of a staffing reserve for implementation of product, technological and governance solutions and claims leadership market positions not only within the team itself, but within the organization as a whole. The direction of the breakthrough is selected by the team and is approved by the organization leaders considering the current development issues [19]. This could be:

- creation of fundamentally new manufacturing systems and technologies;

- adoption of innovative business models;

- digitalization of asset management and cost management systems.

Table 2 outlines the key requirements that a cross-disciplinary team must meet in terms of competencies. 


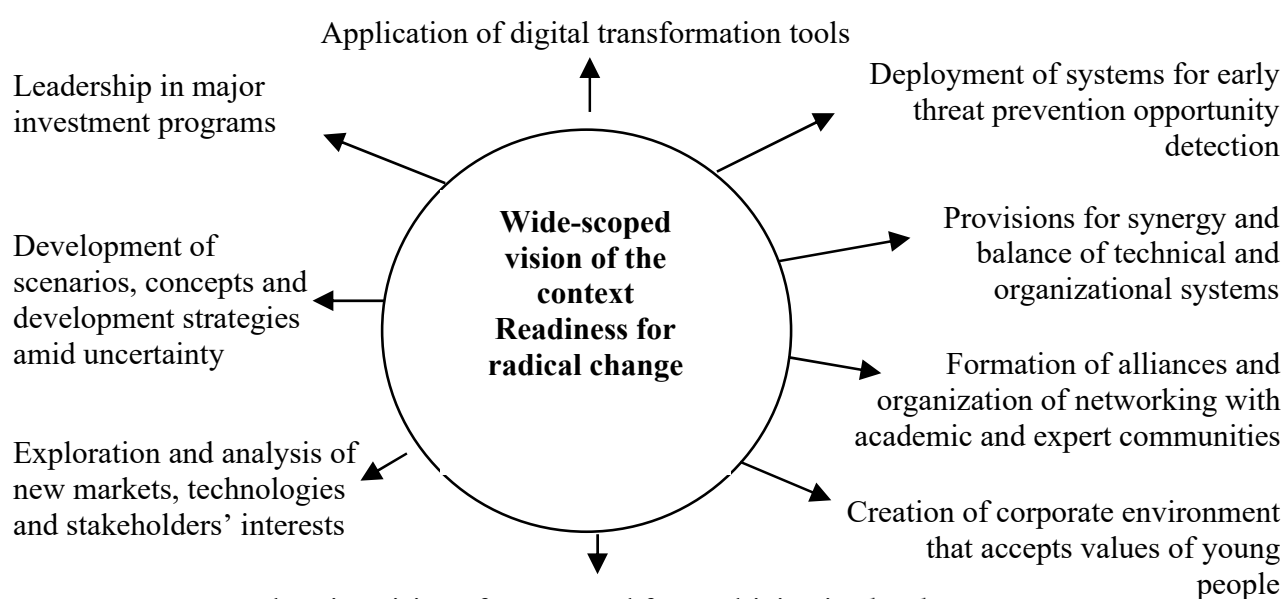

Comprehensive vision of system and forces driving its development people (trends - breakthrough innovations - models of promising markets - intellectual potential of business)

Figure 3: Competencies of a breakthrough team.

Table 2: Competency requirements to be met by breakthrough teams.

\begin{tabular}{|c|c|c|}
\hline Preemptive management & Communications & \\
\hline it) & \multirow{4}{*}{$\begin{array}{l}\text { Building cooperation } \\
\text { with external } \\
\text { organizations, search for } \\
\text { a compromise and } \\
\text { coordination of goals } \\
\text { and joint efforts } \\
\text { Engagement of staff } \\
\text { members in } \\
\text { transformational } \\
\text { projects and appraisal of } \\
\text { each participant's } \\
\text { contribution }\end{array}$} & \multirow{4}{*}{$\begin{array}{l}\text { Learning from } \\
\text { mistakes and } \\
\text { proactively } \\
\text { seeking to } \\
\text { avoid them } \\
\text { Accumulation } \\
\text { and } \\
\text { systematization } \\
\text { of knowledge } \\
\text { and its } \\
\text { incorporation } \\
\text { in work } \\
\text { processes }\end{array}$} \\
\hline m; risk & & \\
\hline ns t thenothen readinecs and adiuct them & & \\
\hline unconventional solutions & & \\
\hline
\end{tabular}

5.1 Principles of competency building for breakthrough teams

During the training period:

- mastering the fundamentals of systems thinking and application of the systems approach to practical tasks;

- $\quad$ shaping and building cross-disciplinary communication skills, team collaboration team for the sake of achieving its goals;

- mastering the principles of modeling complex systems that exist in an evolving environment; 
- $\quad$ studying the experience of transformations (successes and failures); identification of trends, systematization of knowledge and its application to solving practical tasks;

- performance appraisal of the team and its members; assessment of its potential and building of individual development trajectories.

During the period of post-training practice:

- increasing the capacity of tasks handled by the team;

- constant contact with the supervisor;

- regularly performed analysis of results, expansion of knowledge, development of internal standards;

- provisions for professional growth and open communication among team members.

A training program for a breakthrough team typically consists of the following methods and instruments:

1. Theoretical training: the scientific framework of the systems approach: the role of systems science; different points of view on the concept of system; evolution; evolution of the definition of systems engineering; the practical significance of the concept and principles of systems thinking in application to socio-technical systems; models and processes of the lifecycle of systems: general concepts and application fields; characteristics of complex systems, identification of complexity, specific ways of maintaining the viability of complex systems; increasing the adaptability and resilience of a system on the basis of resilience principles; peculiarities of organizational culture supporting resilience; creation and development of systems aimed at continuous change in a turbulent environment (agility);

2. Practical training: hands-on experience in dealing with complexity: (a) identification of types of complexity in a system and its environment; (b) shaping new ways of thinking about complexity that make it possible to use relevant approaches; (c) assessment and sharing of methods that are applied to various types of complexity in different situations; ensuring a system's capability to adapt, thus enabling it to stay operational when problems or opportunities emerge unexpectedly (resilience); identification of factors that require the construction of a change-oriented system (agility); determining what requirements the system must meet and application of the Agile Systems Engineering methodology for designing and developing the system.

In our practice, the concept of training for a breakthrough team is summarized by the formula "Self-diagnosis - self-design - self-fulfillment". Consultants organize and provide methodological support to the process of team improvement and managers' and specialists' "learning by action".

As part of the project (Table 3), the members of the team - managers and engineers - are actively engaged in the identification and systematization of the most urgent problems faced by the company; they work on solutions and innovative development projects. The training program is, therefore, synchronized with the company's innovation activities. The feature of the project is a creative atmosphere in the team that boosts idea generation, conceptual elaboration of ideas, testing and pilot deployments.

Our experience of training breakthrough teams for Russia's major energy companies $\mathrm{T}$ Plus and Bashkirenergo and Ural Federal University proved the effectiveness of the program's orientation towards the development of systems thinking, mastering of the systems approach, systems engineering, methodologies that facilitate the resilience and adaptability 
of manufacturing and managerial systems. According to feedback from the members of the teams, they experienced "a breakthrough in the head" comparable to "brain surgery".

1. an ability to change the mental paradigm thanks to the understanding of fundamental laws of development;

2. a much broader vision of the context;

3. knowledge of the methodology of creating flexibility and resilience mechanisms in complex systems;

4. skills for making complex interdisciplinary decisions;

5. an approach to digitalization as an opportunity for a radical rethinking of business models and the entire business, primarily of customer relationships rather than as mere process automation;

6. optimization of individual elements of the lifecycle of objects (products) as one system.

Table 3: Contents of the project.

\begin{tabular}{|c|c|c|c|}
\hline Phase & Diagnosis & $\begin{array}{l}\text { Increased breakthrough } \\
\text { preparedness }\end{array}$ & Evaluation of results \\
\hline Objective & $\begin{array}{l}\text { Systematize problems } \\
\text { and determine priorities } \\
\text { for solutions }\end{array}$ & $\begin{array}{l}\text { Fulfill training program for } \\
\text { addressing development tasks }\end{array}$ & $\begin{array}{l}\text { Present a } \\
\text { comprehensive vision } \\
\text { of company } \\
\text { development }\end{array}$ \\
\hline Activity & $\begin{array}{l}\text { Pre-project analysis - } \\
\text { self-diagnosis } \\
\text { Strategic session } \\
\text { "Systemic problems of } \\
\text { development } \\
\text { (composition, typology, } \\
\text { priorities)" } \\
\text { Report for top- } \\
\text { management } \\
\text { "Development: } \\
\text { problems, prospects, } \\
\text { action plan" }\end{array}$ & $\begin{array}{l}\text { Training program } \\
\text { 1. Managers and engineers acting } \\
\text { preemptively } \\
\text { 2. Complex project management } \\
\text { 3. Investment management and } \\
\text { asset management } \\
\text { 4. Strengthening of innovation } \\
\text { 5. "A vision of the future that } \\
\text { inspires high performance" } \\
\text { workshop }\end{array}$ & $\begin{array}{l}\text { Conceptual program } \\
\text { "My company is } \\
\text { oriented towards the } \\
\text { future" } \\
\text { - Architecture } \\
\text { - New engineering } \\
\text { - Strategic priorities } \\
\text { - Self-development } \\
\text { mechanism } \\
\text { - Business model }\end{array}$ \\
\hline Focus & $\begin{array}{l}\text { Barriers to increasing } \\
\text { business effectiveness }\end{array}$ & Competencies for a breakthrough & $\begin{array}{l}\text { Parameters of } \\
\text { innovation-oriented } \\
\text { corporate environment }\end{array}$ \\
\hline
\end{tabular}

\section{CONCLUSION}

In this study, the authors place the emphasis on the complexity of systems of differing nature that form the skeleton framework of the digital industry and on systems engineering methodologies that provide opportunities for their creation and development.

Systems engineering methodologies make it possible to find solutions to core problems that emerge in technical as well as in socio-technical systems:

- the problem of a systemic approach - creation of a single information space, support of multi-disciplinarity, knowledge exchange among professional groups, creation of a meta-model of systems throughout the entire lifecycle, filling them with changeable models of components;

- the problem of complexity - introduction of viable technical solutions and principles of creation and support of complex systems (role, responsibility, management tools); 
- human integration into the system - methods and instruments for creating complex systems oriented towards humans acting within the system and using it for reaching their goals;

- the problem of creating viable systems, that is, systems that are adapted to operating in a highly unstable environment and perform critical functions disruptions to which are either unacceptable or too costly.

Systems that operate in such conditions must be capable of changing fast (both reactively and proactively). To equip them with such a capability, architectural patterns, design principles and agility guidelines have been worked out. Additionally, behavioral principles that ensure the implementation of fast and safe transformations have been determined and a model of Agile system lifecycle management has been designed.

\section{ACKNOWLEDGEMENT}

The work was supported by Act 211 of the Government of the Russian Federation, contract number 02.A03.21.0006.

\section{REFERENCES}

[1] Gitelman, L.D., Gavrilova, T.B., Kozhevnikov, M.V. \& Starikov, E.M., Ensuring resilience and agility of complex organizational-technical systems. International Journal of Design and Nature and Ecodynamics, 13(2), pp. 208-220, 2018.

[2] Batovrin, V.K. \& Pozin, B.A., Requirements engineering at the modern enterprise. CEUR Workshop Proceedings, 1989, pp. 380-387, 2017.

[3] ISO/IEC/IEEE 24765:2017, Systems and software engineering: Vocabulary. www.iso.org/standard/71952.html. Accessed on: 22 Aug. 2020.

[4] Gavrilova, T.B., Gitelman, L.D. \& Kozhevnikov, M.V., Systems Engineering for Managers, Economics: Moscow, 2017. (In Russian.)

[5] Dove, R., Response Ability: The Language, Structure, and Culture of the Agile Enterprise, Wiley: New York, 2001.

[6] Dove, R., Fundamental principles for Agile systems engineering. www.parshift.com/ Files/PsiDocs/Rkd050324CserPaper.pdf. Accessed on: 22 Aug. 2020.

[7] Dove, R. \& Turkington, G., Relating Agile development to Agile operations. www.parshift.com/Files/PsiDocs/Pap080404Cser2008DevOpsMigration.pdf.

Accessed on: 22 Aug. 2020.

[8] Dove, R., Engineering Agile systems: Creative-guidance frameworks for requirements and design. www.parshift.com/Files/PsiDocs/Rkd060407CserEngineeringAgile Systems.pdf. Accessed on: 22 Aug. 2020.

[9] Dove, R. \& LaBarge, R., Fundamentals of Agile systems engineering: Parts 1 and 2. www.parshift.com/s/140630IS14-AgileSystemsEngineering-Part1\&2.pdf. Accessed on: 22 Aug. 2020.

[10] Dove, R. \& Schindel, B., Agility in systems engineering: Findings from recent studies. www.parshift.com/s/ASELCM-AgilityInSE-RecentFindings.pdf. Accessed on: 22 Aug. 2020.

[11] Schindel, B. \& Dove, R., Introduction to the Agile Systems engineering life cycle MBSE pattern.

www.parshift.com/s/160718IS16-IntroToTheAgileSystemsEngineeringLifeCycle MBSEPattern.pdf. Accessed on: 22 Aug. 2020.

[12] ISO/IEC/IEEE 15288:2015, Systems and software engineering: System life cycle processes. www.iso.org/ru/standard/63711.html. Accessed on: 22 Aug. 2020. 
[13] Madni, A.M. \& Jackson, S., Towards a conceptual framework for resilience engineering. IEEE Systems Journal, 3(2), pp. 181-191, 2009.

[14] Jackson, S. \& Ferris, T., Resilience principles for engineered systems. Systems Engineering, 16(2), pp. 152-164, 2013.

[15] Woods, D.D., Essential characteristics of resilience. Resilience Engineering: Concepts and Precepts, eds E. Hollnagel, D. Woods \& N. Leveson, Ashgate: Aldershot, UK, pp. 21-34, 2006.

[16] Jackson, S., Resilience principles for the ICT Sector. Critical Information Infrastructure Protection and Resilience in the ICT Sector, eds P. Theron \& S. Bologna, IGI Global: Hershey, pp. 36-49, 2013.

[17] Jackson, S., Architecting Resilient Systems: Accident Avoidance and Survival and Recovery from Disruptions, Wiley: Hoboken, NJ, 2010.

[18] Jackson, S., Erlick, K. \& Gutierrez, J., The science of organizational psychology applied to mission assurance. Conference on Systems Engineering Research (CSER) Proceedings, Los Angeles, CA, 7-8 Apr., Paper 102, 2006.

[19] Gitelman, L., Kozhevnikov, M. \& Ryzhuk, O., Advance management education for power-engineering and industry of the future. Sustainability, 11, p. 5930, 2019. 